\title{
Regional improvements in lumbosacropelvic Hounsfield units following teriparatide treatment
}

\author{
Patrick M. Flanigan, MD, ${ }^{1}$ Anthony L. Mikula, MD, ${ }^{1}$ Pierce A. Peters, MD, ${ }^{1}$ Soliman Oushy, MD, ${ }^{1}$ \\ Jeremy L. Fogelson, MD, ${ }^{1}$ Mohamad Bydon, MD, ${ }^{1}$ Brett A. Freedman, MD, ${ }^{2}$ \\ Arjun S. Sebastian, MD, ${ }^{2}$ Bradford L. Currier, MD, ${ }^{2}$ Ahmad Nassr, MD, ${ }^{2}$ Kurt A. Kennel, MD, ${ }^{3}$ \\ Paul A. Anderson, MD, ${ }^{4}$ David W. Polly, MD, ${ }^{5}$ and Benjamin D. Elder, MD, PhD ${ }^{1,2,6}$
}

\begin{abstract}
Departments of ${ }^{1}$ Neurologic Surgery, ${ }^{2}$ Orthopedic Surgery, and ${ }^{6}$ Biomedical Engineering, Mayo Clinic, Rochester; ${ }^{3}$ Division of Endocrinology, Department of Internal Medicine, Mayo Clinic, Rochester; ${ }^{5}$ Department of Orthopedic Surgery, University of Minnesota, Minneapolis, Minnesota; and ${ }^{4}$ Department of Orthopedics and Rehabilitative Medicine, University of Wisconsin School of Medicine and Public Health, Madison, Wisconsin
\end{abstract}

\begin{abstract}
OBJECTIVE Opportunistic Hounsfield unit (HU) determination from CT imaging has been increasingly used to estimate bone mineral density (BMD) in conjunction with assessments from dual energy $x$-ray absorptiometry (DXA). The authors sought to compare the effect of teriparatide on HUs across different regions in the pelvis, sacrum, and lumbar spine, as a surrogate measure for the effects of teriparatide on lumbosacropelvic instrumentation.
\end{abstract}

METHODS A single-institution retrospective review of patients who had been treated with at least 6 months of teriparatide was performed. All patients had at least baseline DXA as well as pre- and post-teriparatide CT imaging. HUs were measured in the pedicle, lamina, and vertebral body of the lumbar spine, in the sciatic notch, and at the S1 and S2 levels at three different points (ilium, sacral body, and sacral ala).

RESULTS Forty patients with an average age of 67 years underwent a mean of 20 months of teriparatide therapy. Mean HUs of the lumbar lamina, pedicles, and vertebral body were significantly different from each other before teriparatide treatment: $343 \pm 114,219 \pm 89.2$, and $111 \pm 48.1$, respectively ( $<$ 0.001). Mean HUs at the S1 level for the ilium, sacral ala, and sacral body were also significantly different from each other: $124 \pm 90.1,-10.7 \pm 61.9$, and 99.1 \pm 72.1 , respectively $(p<0.001)$. The mean HUs at the S2 level for the ilium and sacral body were not significantly different from each other, although the mean $\mathrm{HU}$ at the sacral ala $(-11.9 \pm 52.6)$ was significantly lower than those at the ilium and sacral body $(p=0.003$ and 0.006 , respectively). HU improvement occurred in most regions following teriparatide treatment. In the lumbar spine, the mean lamina HU increased from 343 to $400(p<0.001)$, the mean pedicle HU increased from 219 to $242(p=0.04)$, and the mean vertebral body HU increased from 111 to $134(p<0.001)$. There were also significant increases in the S1 sacral body (99.1 to 130, $p<0.05)$, S1 ilium (124 vs 165, $p=0.01)$, S1 sacral ala $(-10.7$ vs $3.68, p=$ 0.04 ), and S2 sacral body (168 vs $189, p<0.05)$.

CONCLUSIONS There was significant regional variation in lumbar and sacropelvic HUs, with most regions significantly increasing following teriparatide treatment. The sacropelvic area had lower HU values than the lumbar spine, more regional variation, and a higher degree of correlation with BMD as measured on DXA. While teriparatide treatment resulted in HUs > 110 in the majority of the lumbosacral spine, the HUs in the sacral ala remained suggestive of severe osteoporosis, which may limit the effectiveness of fixation in this region.

https://thejns.org/doi/abs/10.3171/2020.5.FOCUS20273

KEYWORDS Hounsfield units; lumbar; osteoporosis; pelvis; sacrum; teriparatide

$\mathrm{L}$ ow bone mineral density (BMD) may be present in as many as $75 \%$ of patients undergoing spine surgery, with even higher rates in women older than 50 years. ${ }^{1}$ Improving a patient's BMD prior to surgery is recognized as an important factor in preventing postop- erative complications such as pseudarthrosis, junctional kyphosis, pelvic insufficiency fractures below a lumbosacral fusion, ${ }^{2-4}$ and instrumentation failure. ${ }^{5-7}$ Dual-energy $\mathrm{x}$-ray absorptiometry (DXA) is the most commonly utilized method for measuring BMD, but the clinical valida-

ABBREVIATIONS BMD = bone mineral density; DXA = dual-energy x-ray absorptiometry; HU = Hounsfield unit; PTH = parathyroid hormone; ROI = region of interest. SUBMITTED April 1, 2020. ACCEPTED May 13, 2020.

INCLUDE WHEN CITING DOI: 10.3171/2020.5.FOCUS20273. 
tion of this technique has almost exclusively been directed at its ability to assess osteoporotic fracture risk, and it is an imperfect tool. Given the prevalence and morbidity of diminished bone quality in patients undergoing spinal fusion surgery and the limited predictive value of BMD for osteoporosis-related complications of spine surgery, recent work has focused on finding new methods or combinations of methods to better assess bone quality in these patients.

Hounsfield units (HUs) are a relative measurement of $\mathrm{x}$-ray attenuation obtained from routine CT images. Such images are obtained in the vast majority of patients undergoing elective spinal fusion surgery, and HUs have been correlated to BMD assessed by DXA. ${ }^{8-12}$ Thus, HU determination, which can be performed by the surgeon on essentially all PACS systems, represents an opportunistic and essentially costless tool for preoperatively assessing bone quality that has the added benefit of being able to measure more specific regions of interest (ROIs; i.e., vertebral body or pedicle isthmus). An HU cutoff of $<110$ in the lumbar spine has been reported to be indicative of osteoporosis. ${ }^{13,14}$ Given degenerative changes and deformity in the spine, the lumbar BMD should not be used and thus is not available to assess bone health in many spine patients. ${ }^{15}$ Therefore, HU measurements may be a better tool for use in the lumbar spine and may be superior when assessing the risk for vertebral fracture. ${ }^{16}$ HUs allow for more focused assessment of the trabecular bone based on a defined ROI and eliminate the cortical bone, sclerotic changes, and posterior element bone that may affect DXA measurements. ${ }^{17,18}$ However, HUs and spine and hip DXA have been used as complementary or standalone methods to evaluate bone quality. ${ }^{10,19}$

Teriparatide, a parathyroid hormone (PTH) analog (PTH 1-34), is an anabolic medication that promotes differentiation of osteoblasts, resulting in new bone formation, and is currently approved for the treatment of osteoporosis. Many spine surgeons, often working in consort with bone health specialists such as endocrinologists, have used teriparatide in an off-label fashion to mitigate poor bone quality prior to elective spine surgery. Although there is evidence that antiresorptive therapies may be beneficial for improving fusion rates and decreasing subsequent vertebral column fractures, ${ }^{20}$ there is limited evidence that teriparatide is superior to bisphosphonates in promoting fusion and reducing pedicle screw loosening., ${ }^{7,21}$ Additionally, in prior studies, a longer period of teriparatide administration (i.e., at least 6 months) resulted in a faster time to fusion and increased fusion rate, ${ }^{22}$ while improving bone strength and reducing fracture risk in postmenopausal women. ${ }^{23,24}$ We have previously demonstrated that teriparatide results in an increase in HUs of the lumbar spine out of proportion to the improvement seen in the DXA Tscore. ${ }^{25}$ Typically, teriparatide is just one pharmacological piece of an overall comprehensive strategy used to avoid construct failure.

Investigating the effects of teriparatide on bone density with attention to region-specific variation may provide information on optimizing lumbosacropelvic fixation. For instance, Klineberg et al. ${ }^{2}$ reported a 3.1\% rate of sacral insufficiency fractures following long-segment fusion, as well as two-thirds of patients with fractures requiring surgical revision. Similarly, Odate et al. ${ }^{3}$ reported a $4.3 \%$ fracture rate, and there is an increased risk with sagittal imbalance, a high pelvic incidence, obesity, and osteoporosis. ${ }^{3,4}$ The sacropelvic area is of particular interest in that sacropelvic fixation has been considered essential to optimize construction stability for long-segment constructs involving the lumbar spine. ${ }^{26}$ In a randomized trial of patients with symptomatic pelvic insufficiency fractures, Peichl et al. reported that PTH 1-84 significantly reduced pain, improving walking ability and bony healing compared to placebo..$^{27,28}$ Thus, the use of such an anabolic medication may be useful to prevent and treat such complications related to spinal surgery. With the renewed attention on sacroiliac joint fixation and fusion, it is important to better understand the regional variation in bone density in the sacrum and ilium and any potential benefits of teriparatide.

We hypothesized that a minimum of 6 months of teriparatide treatment would result in significant improvement in the regional bone density of the lumbosacral spine and ilium, using HUs as a surrogate for BMD. Therefore, the objective of this study was to compare the effect of teriparatide on HUs across different regions in the lumbar spine and sacropelvic region. Secondly, we correlated HU changes at each spinal level and anatomical bony region with DXA BMD.

\section{Methods}

After obtaining institutional review board approval, we performed a retrospective review of records dated 20022018 across all campuses at a single multicenter institution. We identified patients who had been prescribed teriparatide, had both pre- and posttreatment DXA, and had pre- and posttreatment abdominal or lumbar spine CT, including the pelvis. Inclusion criteria were treatment with at least 6 months of teriparatide and CT encompassing all spinal ROIs both before and after treatment. The pre- and posttreatment CT scans had been obtained within a 3-year period. Collected data included age, height, weight, sex, BMI, osteoporosis medications, smoking status, metabolic laboratory tests, hemodialysis status, duration of teriparatide treatment, time between $\mathrm{CT}$ scans, time between DXA scans, and BMD at various anatomical regions on DXA. We also manually measured HUs in various ROIs on the CT. HUs were measured using ROI circles drawn in accordance with a previously reported methodology. ${ }^{10}$ Two independent reviewers performed all HU measurements.

\section{HU Measurements}

For each patient, the following measurements were made using axial scans (Fig. 1) for the lumbar vertebrae: laminar cancellous bone ( 2 sides, 5 levels), pedicles (2 sides, 5 levels), and vertebral body ( 3 measurements averaged, 5 levels). The following measurements were made at S1-2 using axial scans: ilium (2 sides, 2 levels), sacral ala ( 2 sides, 2 levels), and sacral body (2 levels; Fig. 2). Finally, coronal CT images were utilized to measure the sciatic notches ( 2 sides). When measuring the vertebral body, three ROIs (just inferior to the superior endplate, mid, and just superior to the inferior endplate) were averaged. Mea- 

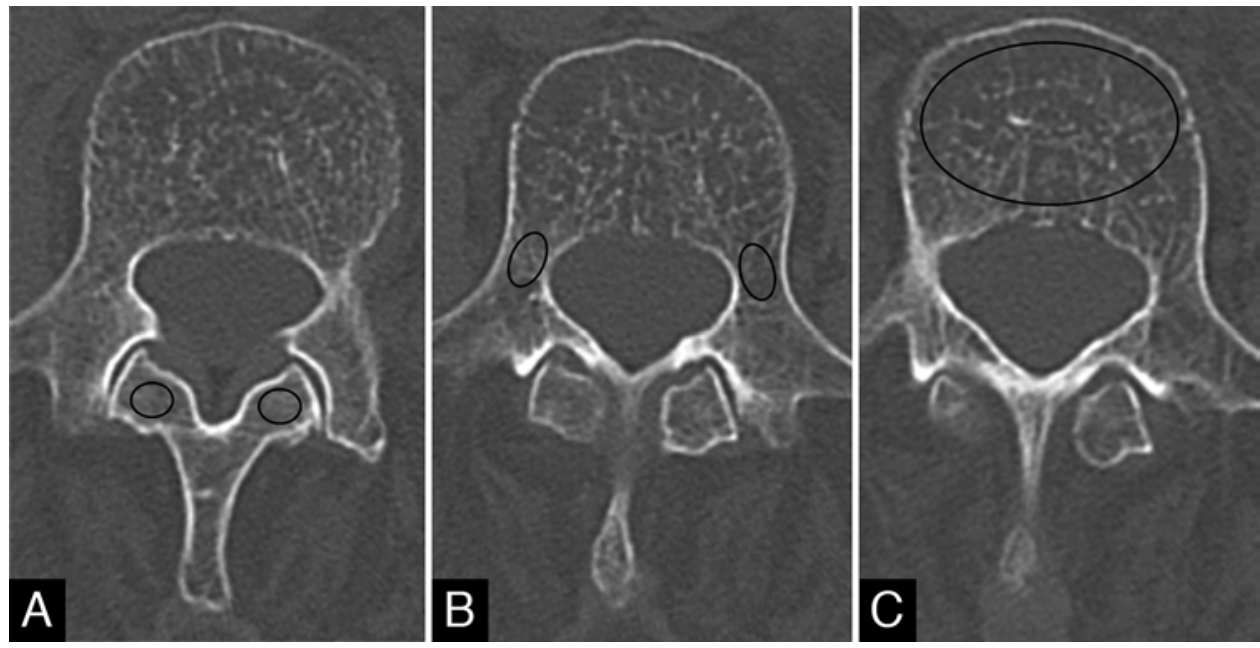

FIG. 1. Representative axial CT scans showing three ROls in the lumbar spine: laminae (A), pedicles (B), and vertebral body (C).

surements of the iliac crest were made via bilateral ROI ellipses within the trabecular bone at the S2 level. In the lumbar spine, the values from each level were combined to determine a mean value for the vertebral body, pedicle, and lamina. We excluded measurements of vertebrae containing instrumentation, adjacent to interbody grafts, or with vertebroplasty cement, as the artifact confounded HU measurement.

\section{Statistical Analysis}

Means were compared via two-sample t-tests, paired t-tests, and one-way ANOVA, with statistical significance defined as $p<0.05$. Pearson correlation coefficients were calculated to assess the correlation between HUs and DXA BMD before and after teriparatide therapy at each region (lumbar spine and sacropelvic area). Two independent reviewers performed $\mathrm{HU}$ measurements, and intraclass correlation coefficient calculations were used to determine interrater reliability. Analyses were performed using statistical software (JMP Pro 14.0.0, SAS Institute Inc.).

\section{Results}

\section{Patient Cohort}

We identified a total of 5487 patients who had been prescribed teriparatide over the study period, and 403 of these patients had undergone DXA scanning and lumbar CT imaging. Out of those patients, only 52 had additional CT imaging after 6 months of teriparatide treatment, and 40 patients had imaging adequately encompassing the sacrum and pelvis and were included in the present study. There were 6 men and 34 women with a mean age of 67 \pm 12 years (mean $\pm \mathrm{SD})$. The mean teriparatide treatment duration was $20 \pm 6$ months (Table 1). The mean BMI was $24 \pm 6 \mathrm{~kg} / \mathrm{m}^{2}$. The mean femoral neck, lumbar spine, and total hip DXA T-scores were $-2.6 \pm 0.9,-2.4 \pm 1.5$, and $-2.2 \pm 1.1$, respectively.

\section{HU Regional Variability Prior to Teriparatide Administration}

There were significant differences in HU intensity among regions in the lumbar spine with the lumbar vertebral body, lamina, and pedicle having $111 \pm 48.1,343 \pm$ 114 , and $219 \pm 89.2 \mathrm{HU}$, respectively ( $<0.001$; Table 2). There were significant differences among regions at the S1 level, with the sacral body, ilium, and sacral ala having 99.1 $\pm 72.1,124 \pm 90.1$, and $-10.7 \pm 61.9 \mathrm{HU}$, respectively ( $\mathrm{p}<$ 0.001 ). There were significant differences among regions at the $\mathrm{S} 2$ level, with the sacral body and ilium having HUs of $168 \pm 55.4$ and $92.8 \pm 105$, respectively, while the sacral ala
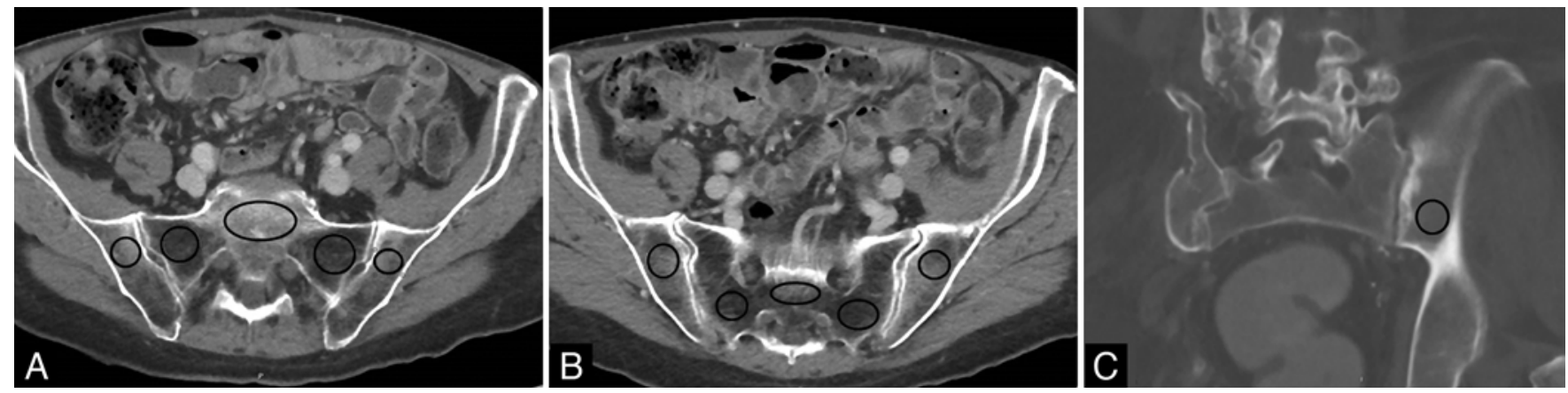

FIG. 2. Representative axial CT scans of the S1 level (A), S2 level (B), and sciatic notch (C). HUs were measured at three different locations at each level: ilium, sacral ala, and sacral body. 
TABLE 1. Overall characteristics of 40 patients receiving teriparatide treatment

\begin{tabular}{lc}
\hline & Value \\
\hline Average age in yrs (range) & $67 \pm 12(36-84)$ \\
\hline Sex: F/M & $34: 6$ \\
\hline Mean height in cm & $161 \pm 8$ \\
\hline Mean weight in kg & $62 \pm 15$ \\
\hline Mean BMl in kg/m² & $24 \pm 6$ \\
\hline $\begin{array}{l}\text { Mean duration of teriparatide therapy in mos } \\
\text { (range) }\end{array}$ & $20 \pm 6(6-27)$ \\
\hline Average time btwn pre \& post CT scans in mos & $39 \pm 21$ \\
\hline Average time btwn pre \& post DXA in mos & $28 \pm 13$ \\
\hline Baseline mean DXA T-score at femoral neck & $-2.6 \pm 0.9$ \\
\hline Baseline mean DXA T-score at lumbar spine & $-2.4 \pm 1.5$ \\
\hline Baseline mean DXA T-score at total hip & $-2.2 \pm 1.1$ \\
\hline
\end{tabular}

Values are expressed as the mean \pm SD, unless indicated otherwise.

was significantly lower at $-11.9 \pm 52.6 \mathrm{HU}(\mathrm{p}<0.001)$. The sciatic notch was a mean of $30.6 \pm 65.0 \mathrm{HU}$.

\section{HU Changes After Teriparatide Administration}

There was a significant increase in all three lumbar regions following teriparatide administration. The lumbar lamina HU increased $16.4 \%$ on average (343 to 400 , p < $0.001)$. The lumbar pedicles HU increased $10.2 \%$ on average (219 to $242, \mathrm{p}=0.04)$, and the lumbar vertebral body HU increased 20.7\% (111 to 134, p < 0.001; Table 2 and Fig. 3A).

At $\mathrm{S} 1$, there was a significant increase in the HUs of the sacral ala $(-10.7$ to $3.68, p=0.04)$, the ilium $(124$ to $165, p$ $=0.01)$, and the sacral body (99.1 to $130, \mathrm{p}<0.05$; Table 2 and Fig. 3B). At S2, there was no significant change in the HUs of the sacral ala $(-11.9$ to $-8.43, p=0.54)$ or the ilium (92.8 to $112, \mathrm{p}=0.19)$; however, the HUs of the sacral body significantly increased (168 to $189, \mathrm{p}<0.05)$. The
HUs at the sciatic notch had a significant $86.7 \%$ increase after teriparatide treatment (30.6 to 57.1, $\mathrm{p}=0.004$ ).

\section{DXA BMD}

Based on DXA, femoral neck BMD $\left(\mathrm{g} / \mathrm{cm}^{2}\right)$ increased by $3 \%(0.65$ to $0.66, \mathrm{p}=0.07)$, lumbar $\mathrm{BMD}$ increased by $10 \%(0.83$ to $0.92, \mathrm{p}<0.001)$, and total hip BMD increased by $5 \%(0.72$ to $0.75, \mathrm{p}=0.001)$.

\section{HU Correlation to DXA BMD}

Prior to teriparatide treatment, the Pearson correlation coefficients between lumbar spine HUs and femoral neck BMD, lumbar BMD, or total hip BMD were 0.27 (25th75th IQR -0.14 to $0.61, \mathrm{p}=0.20$ ), 0.45 (IQR 0.10 to 0.69 , $\mathrm{p}$ $=0.01)$, and $0.46(\mathrm{IQR}-0.03$ to $0.77, \mathrm{p}=0.06)$, respectively (Table 3). The Pearson correlation coefficients between sacropelvic area HUs and the femoral neck BMD, lumbar BMD, or total hip BMD were 0.42 (IQR 0.02 to $0.71, \mathrm{p}=$ 0.04 ), 0.38 (IQR 0.03 to $0.65, \mathrm{p}=0.04$ ), and 0.49 (IQR 0.02 to $0.79, \mathrm{p}<0.05)$, respectively.

Following teriparatide treatment, the Pearson correlation coefficients between lumbar spine HUs and femoral neck BMD, lumbar BMD, or total hip BMD were 0.37 (IQR -0.03 to $0.67, \mathrm{p}=0.07), 0.43$ (IQR 0.07 to $0.69, \mathrm{p}=$ 0.02 ), and 0.51 (IQR 0.02 to $0.80, \mathrm{p}=0.04$ ), respectively. The Pearson correlation coefficients between sacropelvic area HUs and femoral neck BMD, lumbar BMD, or total hip BMD were 0.55 (IQR 0.20 to $0.78, \mathrm{p}=0.004$ ), 0.39 (IQR 0.02 to $0.67, \mathrm{p}=0.04$ ), and 0.66 (IQR 0.25 to 0.87 , p $=0.005)$, respectively. Figure 4 demonstrates representative correlations between lumbar DXA BMD and lumbar HUs and between total hip DXA BMD and sacropelvic HUs, both following treatment with teriparatide, with the highest correlation between total hip DXA and sacropelvic HUs.

\section{Reliability}

Overall, the HU measurements had an interrater reliability of 0.88 ( $\mathrm{p}<0.0001)$. Lumbar spine measurements had an interrater reliability of 0.99 ( $p<0.0001)$, while

TABLE 2. Regional HU values pre- and posttreatment with teriparatide

\begin{tabular}{lcccc}
\hline \multicolumn{1}{c}{ Region } & Pretreatment HUs & Posttreatment HUs & \% Change & p Value \\
\hline Lumbar lamina & 343 & 400 & 16.4 & $<0.001$ \\
\hline Lumbar pedicle & 219 & 242 & 10.2 & 0.04 \\
\hline Lumbar vertebral body & 111 & 134 & 20.7 & $<0.001$ \\
\hline S1 ilium & 124 & 165 & 33.1 & 0.01 \\
\hline S1 sacral ala & -10.7 & 3.68 & $\mathrm{NA}$ & $\mathbf{0 . 0 4}$ \\
\hline S1 vertebral body & 99.1 & 130 & 23.8 & $<0.05$ \\
\hline S2 ilium & 92.8 & 112 & 20.7 & 0.19 \\
\hline S2 sacral ala & -11.9 & -8.43 & $\mathrm{NA}$ & 0.54 \\
\hline S2 vertebral body & 168 & 189 & 12.5 & $<0.05$ \\
\hline Sciatic notch & 30.6 & 57.1 & 86.7 & $\mathbf{0 . 0 0 4}$ \\
\hline
\end{tabular}

$\mathrm{NA}=$ not available, written when either $\mathrm{HU}$ value was negative, as this made assessment of percent change difficult to interpret.

Boldface type indicates statistical significance. 


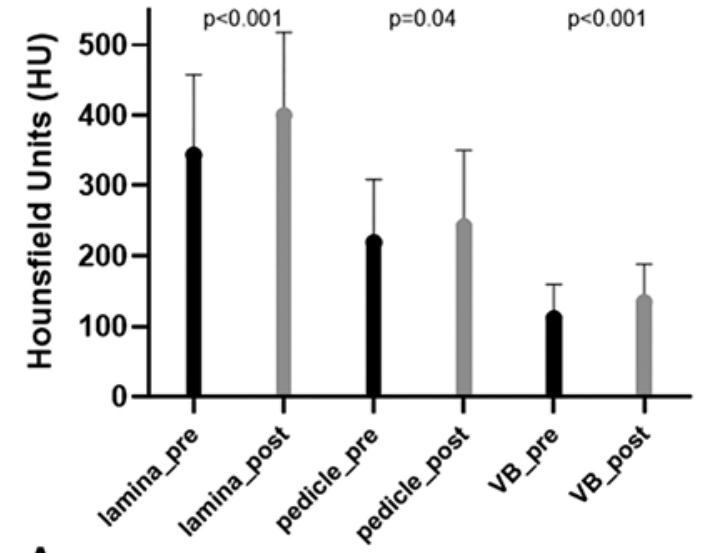

A

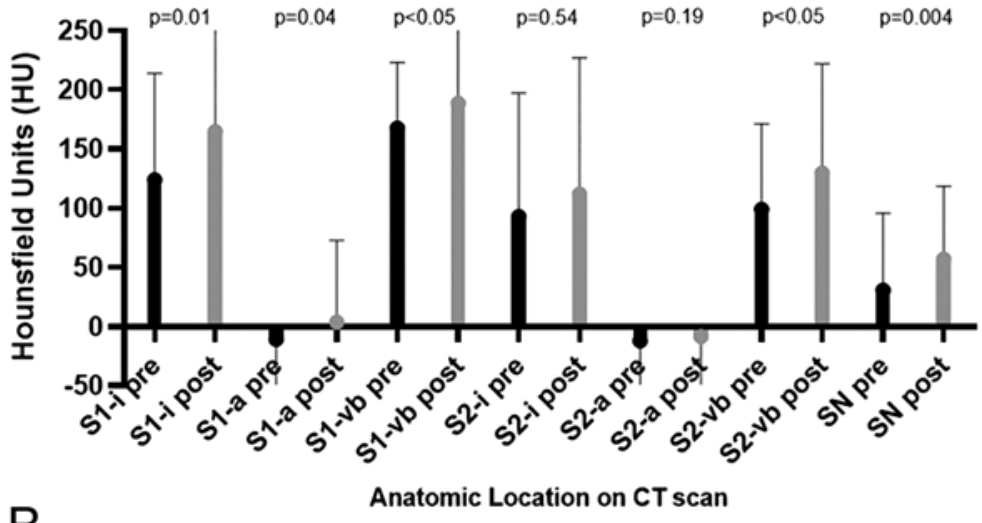

B

FIG. 3. A: Increase in HUs following teriparatide treatment averaged across lumbar levels at the lamina, pedicle, and vertebral body (VB). B: Change in HUs following teriparatide treatment in the ilium (i), sacral ala (a), and vertebral body (vb) at the S1 and S2 levels, as well as the sciatic notch (SN). pre = pretreatment; post $=$ posttreatment.

sacropelvic measurements had an interrater reliability of $0.78(\mathrm{p}<0.0001)$.

\section{Discussion}

In our study, teriparatide increased average $\mathrm{HU}$ values across all regions of the lumbar spine and most regions in the sacropelvic area. Additionally, we noted that compared to the other regions, the vertebral bodies of the lumbosacral vertebra showed the most consistent and most robust response to teriparatide. In osteoporosis, there is a loss of overall trabecular bone mass, with a reduced trabecular bone volume fraction and lower trabecular number. ${ }^{29}$ However, it is unknown why certain bony regions may exhibit a differential response to anabolic agents. ${ }^{30}$

In our study, the more consistent and seemingly larger increase in response to teriparatide at the vertebral body likely results from the increased ratio of trabecular bone relative to cortical bone in the vertebral body, as teriparatide has a greater effect on trabecular bone than cortical bone. ${ }^{31-33}$ For instance, both Genant et al. ${ }^{32}$ and Borggrefe et al. ${ }^{31}$ reported an increase in femoral trabecular volumetric BMD (vBMD) with a decrease in proximal femoral cortical vBMD at 12 and 24 months, respectively. There is an early transient increase in femoral cortical bone turnover and cortical porosity following initiation of teripara- tide therapy, though this may not decrease bone strength. ${ }^{34}$ Additionally, there is evidence that loading through exercise may augment the response to teriparatide,,$^{35}$ and it has been proposed that an increased catabolic effect of teriparatide on cortical porosity at the tibia and radius may result from different loading conditions. ${ }^{33}$

Additionally, preclinical and limited clinical data suggest that teriparatide has the potential to increase spinal fusion rates and decrease pseudarthrosis rates.,21,22,36 For instance, in a rabbit posterolateral fusion model, increasing doses of teriparatide up to $40 \mu \mathrm{g} / \mathrm{kg} /$ day resulted in an increased fusion mass volume as compared to that in the autograft-alone control group..$^{36}$ In a clinical study, Ohtori et al. found that a daily injection of teriparatide following lumbar fusion in osteoporotic women resulted in a significantly lower rate of pedicle screw loosening compared to that in patients taking risedronate and in controls. In a follow-up study, the same group reported an increased fusion rate as well as a faster time to fusion. ${ }^{22}$ Similarly, Ebata et $a l .{ }^{28}$ found that weekly teriparatide administration following lumbar interbody fusion surgery in patients with osteoporosis increased bone growth within the fusion site at even 4 months postoperatively. Other groups have reported an increased rate of pelvic fracture healing and improved function in elderly osteoporotic women following only 8 weeks of PTH treatment. ${ }^{27}$ Therefore, it is

TABLE 3. HU correlation with DXA BMD before and after treatment with teriparatide

\begin{tabular}{|c|c|c|c|}
\hline \multirow[b]{2}{*}{ Level } & \multicolumn{3}{|c|}{ Pearson Correlation Coefficient (IQR, p value) } \\
\hline & Femoral Neck & Lumbar & Total Hip \\
\hline Lumbar before & $0.27(-0.14$ to $0.61,0.20)$ & $0.45(0.10$ to $0.69,0.01)$ & $0.46(-0.03$ to $0.77,0.06)$ \\
\hline Lumbar after & $0.37(-0.03$ to $0.67,0.07)$ & $0.43(0.07$ to $0.69,0.02)$ & $0.51(0.02$ to $0.80,0.04)$ \\
\hline $\begin{array}{l}\text { Sacropelvic region } \\
\text { before }\end{array}$ & $0.42(0.02$ to $0.71,0.04)$ & $0.38(0.03$ to $0.65,0.04)$ & $0.49(0.02$ to $0.79,<0.05)$ \\
\hline $\begin{array}{l}\text { Sacropelvic region } \\
\text { after }\end{array}$ & $0.55(0.20$ to $0.78,0.004)$ & $0.39(0.02$ to $0.67,0.04)$ & $0.66(0.25$ to $0.87,0.005)$ \\
\hline
\end{tabular}

Boldface type indicates statistical significance. 


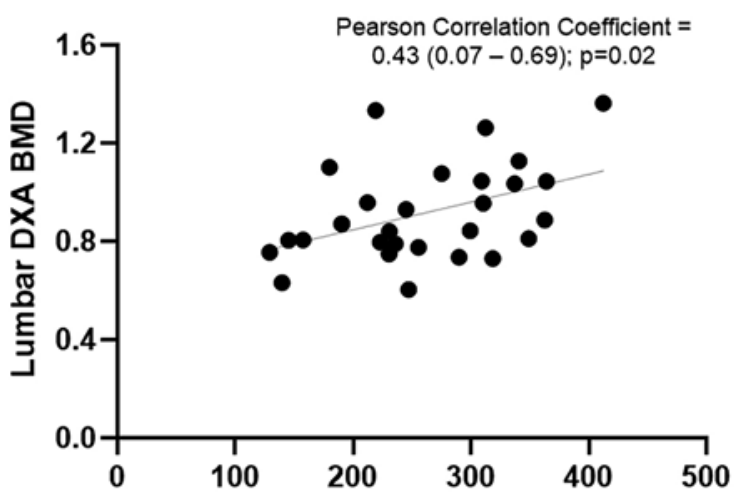

A

Lumbar Hounsfield Units

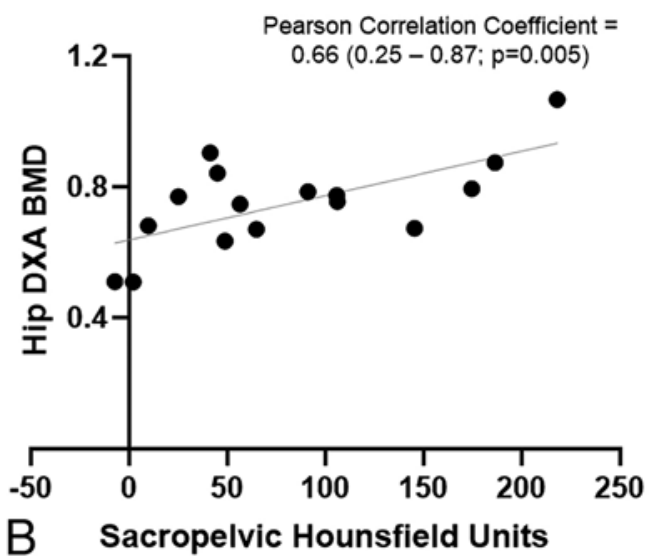

FIG. 4. A: Correlation between lumbar DXA BMD after teriparatide treatment and lumbar HUs following teriparatide treatment. B: Correlation between total hip DXA BMD following teriparatide treatment and sacropelvic HUs following teriparatide treatment.

important to better define how teriparatide's impact can be optimized for a specific surgery involving a given region.

A significant degree of regional variation in HUs was observed, especially in the sacrum, where the sacral ala had the worst bone density with negative HU measurements. The negative values suggest red marrow loss with large voids in trabeculae and/or fatty infiltration of the bone. These results were similar to those of a prior study on a general population, in which the central sacral ala had the lowest HU measurements with a mean of 24, while the L1 and S1 vertebral bodies were 165 and 224 HUs, respectively. There was a clear discrepancy in measurements in the sacropelvic area and the lumbar spine overall, with the lumbar spine having significantly higher measurements overall, suggestive of higher BMD. However, in contrast to a prior study assessing sacral HUs,${ }^{37}$ we did not find the S2 level to have less dense bone than the S1 level, which was potentially attributable to differences in populations, with our population being significantly more osteoporotic.

The sacral ala at both S1 and S2 levels had by far the worst baseline HUs, with even negative measurements, and there was no significant improvement in the HUs of the ala and ilium at the S2 level. This result may be attributable to the relatively small sample size and may suggest that teriparatide administration has limited effects at these regions or that these regions had such poor baseline bone density with significant fatty infiltration that even 6 months of teriparatide treatment was not adequate to obtain meaningful improvement. Since both the lumbar spine and pelvis have large stores of cancellous bone, it is still unknown what caused the regional variation detected between the spine and pelvis at baseline. Since reciprocal work has not been performed in subjects with normal bone density, it is not known if this difference is intrinsic or an effect of differential bone loss due to osteoporosis that is greater in the sacrum than in the low lumbar spine. However, these observations are consistent with the fact that the low lumbar spine is less affected by fragility fractures than more cephalad levels, while the sacrum is a common site of insufficiency fractures.

When considering pelvic fixation with S2 alar-iliac (S2AI) screws, our results indicate that even following te- riparatide treatment in osteoporotic patients, there may be minimal biomechanical contribution from the sacral fixation with limited benefit on pullout strength, which is integral to durable sacropelvic fixation. ${ }^{38,39}$ Additionally, with a renewed focus on sacroiliac fixation and fusion, these results suggest that fixation in the sacral ala may be limited in the osteoporotic patient population. Alternatively, the findings may emphasize the importance of obtaining bicortical or tricortical fixation of sacral pedicle screws in this population given that the most marked improvements in HUs occurred at the vertebral body. ${ }^{26,40}$ Overall, given the poor bone density in the cancellous bone of the pelvis, trajectories such as S2AI screws that involve multiple cortical surfaces may be superior in osteoporotic patients. However, further biomechanical studies are required to better understand the implications of this regional heterogeneity. To our knowledge, this is one of few studies analyzing changes in HUs throughout the lumbosacral spine and pelvis following teriparatide therapy. Our focus on region-specific changes may better elucidate the mechanistic effects of at least 6 months of perioperative teriparatide administration and eventually permit patient-specific screw trajectory or construct considerations.

Improvement in the BMD was significant on DXA of the femoral neck, lumbar spine, and hip regions, with posttreatment increases of 3\%,10\%, and 5\%, respectively. However, there was a low to moderate degree of correlation between baseline HUs and DXA BMD, as well as the improvements observed in HUs and DXA BMD. The correlation was stronger after teriparatide administration and tended to be stronger in the sacropelvic region than in the lumbar region, though these were still relatively moderate correlations. Consistent with our recently published findings on the lumbar vertebral body alone, ${ }^{25}$ the increase in HUs we detected in this study was, on a percentage basis, out of proportion to the increase observed on DXA scans. ${ }^{34,41,42}$ This may suggest that $\mathrm{HU}$ analysis is more sensitive than DXA scans when assessing teriparatiderelated changes to bone density, likely since HU measurements allow for more region-specific measurements than DXA scans, with less interference from degenerative pathology of the spine. There is further support for the use of 
CT-based measurements as opposed to DXA, as Graeff et al. ${ }^{43}$ found that quantitative CT was better associated with fracture status and severity than DXA measurements at the spine or hip.

There are several limitations to this study, including its retrospective nature and small sample size. Additionally, as with most studies on HU assessments, there can be differences in the measurements based on the use of different CT machines and varied distances from the patient. Furthermore, there can be significant bone loss following the completion of teriparatide treatment, with a recommendation for transitioning to bisphosphonate therapy. Since we did not make all the posttreatment HU measurements while patients were still on teriparatide therapy, it is possible that some of the posttreatment measurements would be artificially low. We were not equipped to determine the optimal duration of teriparatide treatment in this study because of our small sample size and the variable amount of time between cessation of teriparatide treatment and reimaging with CT scans. However, we generally recommend at least 6 months of teriparatide preoperatively with postoperative continuation to complete the full 24 months of treatment.7,21,22,28,31 Finally, we realize that BMD assessed by any technique is merely a proxy for bone strength and bony fusion potential; therefore, further studies correlating higher HU values and improved clinical outcomes are needed.

\section{Conclusions}

There was significant regional variation in lumbar and sacropelvic HUs, with most regions significantly increasing after at least 6 months of teriparatide treatment. The sacropelvic area had lower HU values-with even negative values of HUs in the sacral ala-more regional variation, and a higher degree of correlation with BMD as measured on DXA. While teriparatide treatment resulted in HUs > 110 in most of the lumbosacral spine, the sacral ala remained with negative HUs suggestive of severe osteoporosis, which may limit the effectiveness of fixation in this region.

\section{References}

1. Chin DK, Park JY, Yoon YS, et al. Prevalence of osteoporosis in patients requiring spine surgery: incidence and significance of osteoporosis in spine disease. Osteoporos Int. 2007; 18(9):1219-1224.

2. Klineberg E, McHenry T, Bellabarba C, et al. Sacral insufficiency fractures caudal to instrumented posterior lumbosacral arthrodesis. Spine (Phila Pa 1976). 2008;33(16): 1806-1811.

3. Odate S, Shikata J, Kimura H, Soeda T. Sacral fracture after instrumented lumbosacral fusion: analysis of risk factors from spinopelvic parameters. Spine (Phila Pa 1976). 2013; 38(4):E223-E229.

4. Papadopoulos EC, Cammisa FP Jr, Girardi FP. Sacral fractures complicating thoracolumbar fusion to the sacrum. Spine (Phila Pa 1976). 2008;33(19):E699-E707.

5. Anderson PA, Jeray KJ, Lane JM, Binkley NC. Bone health optimization: beyond own the bone: AOA critical issues. $J$ Bone Joint Surg Am. 2019;101(15):1413-1419.

6. Etebar S, Cahill DW. Risk factors for adjacent-segment failure following lumbar fixation with rigid instrumentation for degenerative instability. J Neurosurg. 1999;90(2)(suppl): $163-169$.

7. Ohtori S, Inoue G, Orita S, et al. Comparison of teriparatide and bisphosphonate treatment to reduce pedicle screw loosening after lumbar spinal fusion surgery in postmenopausal women with osteoporosis from a bone quality perspective. Spine (Phila Pa 1976). 2013;38(8):E487-E492.

8. Anderson PA, Morgan SL, Krueger D, et al. Use of bone health evaluation in orthopedic surgery: 2019 ISCD official position. J Clin Densitom. 2019;22(4):517-543.

9. Schreiber JJ, Anderson PA, Hsu WK. Use of computed tomography for assessing bone mineral density. Neurosurg Focus. 2014;37(1):E4.

10. Schreiber JJ, Anderson PA, Rosas HG, et al. Hounsfield units for assessing bone mineral density and strength: a tool for osteoporosis management. J Bone Joint Surg Am. 2011;93(11): 1057-1063.

11. Zou D, Li W, Deng C, et al. The use of CT Hounsfield unit values to identify the undiagnosed spinal osteoporosis in patients with lumbar degenerative diseases. Eur Spine J. 2019; 28(8):1758-1766.

12. Pickhardt PJ, Pooler BD, Lauder T, et al. Opportunistic screening for osteoporosis using abdominal computed tomography scans obtained for other indications. Ann Intern Med. 2013;158(8):588-595.

13. Lee SJ, Binkley N, Lubner MG, et al. Opportunistic screening for osteoporosis using the sagittal reconstruction from routine abdominal $\mathrm{CT}$ for combined assessment of vertebral fractures and density. Osteoporos Int. 2016;27(3):1131-1136.

14. Anderson PA, Polly DW, Binkley NC, Pickhardt PJ. Clinical use of opportunistic computed tomography screening for osteoporosis. J Bone Joint Surg Am. 2018;100(23):2073-2081.

15. Lewiecki EM, Binkley N, Morgan SL, et al. Best practices for dual-energy x-ray absorptiometry measurement and reporting: International Society for Clinical Densitometry guidance. J Clin Densitom. 2016;19(2):127-140.

16. Engelke K, Adams JE, Armbrecht G, et al. Clinical use of quantitative computed tomography and peripheral quantitative computed tomography in the management of osteoporosis in adults: the 2007 ISCD Official Positions. J Clin Densitom. 2008;11(1):123-162.

17. Perilli E, Briggs AM, Kantor S, et al. Failure strength of human vertebrae: prediction using bone mineral density measured by DXA and bone volume by micro-CT. Bone. 2012; 50(6):1416-1425.

18. Bolotin HH. DXA in vivo BMD methodology: an erroneous and misleading research and clinical gauge of bone mineral status, bone fragility, and bone remodelling. Bone. 2007; 41(1):138-154.

19. Hendrickson NR, Pickhardt PJ, Del Rio AM, et al. Bone mineral density T-scores derived from CT attenuation numbers (Hounsfield units): clinical utility and correlation with dualenergy x-ray absorptiometry. Iowa Orthop J. 2018;38:25-31.

20. Liu WB, Zhao WT, Shen P, Zhang FJ. The effects of bisphosphonates on osteoporotic patients after lumbar fusion: a meta-analysis. Drug Des Devel Ther. 2018;12:2233-2240.

21. Kim JW, Park SW, Kim YB, Ko MJ. The effect of postoperative use of teriparatide reducing screw loosening in osteoporotic patients. J Korean Neurosurg Soc. 2018;61(4):494-502.

22. Ohtori S, Orita S, Yamauchi K, et al. More than 6 months of teriparatide treatment was more effective for bone union than shorter treatment following lumbar posterolateral fusion surgery. Asian Spine J. 2015;9(4):573-580.

23. Kendler DL, Marin F, Zerbini CAF, et al. Effects of teriparatide and risedronate on new fractures in post-menopausal women with severe osteoporosis (VERO): a multicentre, double-blind, double-dummy, randomised controlled trial. Lancet. 2018;391(10117):230-240. 
24. McClung MR, Grauer A, Boonen S, et al. Romosozumab in postmenopausal women with low bone mineral density. $N$ Engl J Med. 2014;370(5):412-420.

25. Mikula AL, Puffer RC, Jeor JDS, et al. Teriparatide treatment increases Hounsfield units in the lumbar spine out of proportion to DEXA changes. J Neurosurg Spine. 2020;32(1): $50-55$.

26. Lombardi JM, Shillingford JN, Lenke LG, Lehman RA. Sacropelvic fixation: when, why, how? Neurosurg Clin N Am. 2018;29(3):389-397.

27. Peichl P, Holzer LA, Maier R, Holzer G. Parathyroid hormone 1-84 accelerates fracture-healing in pubic bones of elderly osteoporotic women. J Bone Joint Surg Am. 2011; 93(17):1583-1587.

28. Ebata S, Takahashi J, Hasegawa T, et al. Role of weekly teriparatide administration in osseous union enhancement within six months after posterior or transforaminal lumbar interbody fusion for osteoporosis-associated lumbar degenerative disorders: a multicenter, prospective randomized study. $J$ Bone Joint Surg Am. 2017;99(5):365-372.

29. Chen H, Zhou X, Fujita H, et al. Age-related changes in trabecular and cortical bone microstructure. Int J Endocrinol. 2013;2013:213234.

30. Jiang Y, Zhao JJ, Mitlak BH, et al. Recombinant human parathyroid hormone (1-34) [teriparatide] improves both cortical and cancellous bone structure. J Bone Miner Res. 2003; 18(11):1932-1941.

31. Borggrefe J, Graeff C, Nickelsen TN, et al. Quantitative computed tomographic assessment of the effects of 24 months of teriparatide treatment on 3D femoral neck bone distribution, geometry, and bone strength: results from the EUROFORS study. J Bone Miner Res. 2010;25(3):472-481.

32. Genant HK, Engelke K, Bolognese MA, et al. Effects of romosozumab compared with teriparatide on bone density and mass at the spine and hip in postmenopausal women with low bone mass. J Bone Miner Res. 2017;32(1):181-187.

33. Paggiosi MA, Yang L, Blackwell D, et al. Teriparatide treatment exerts differential effects on the central and peripheral skeleton: results from the MOAT study. Osteoporos Int. 2018; 29(6):1367-1378.

34. Keaveny TM, Crittenden DB, Bolognese MA, et al. Greater gains in spine and hip strength for romosozumab compared with teriparatide in postmenopausal women with low bone mass. J Bone Miner Res. 2017;32(9):1956-1962.

35. Jepsen DB, Ryg J, Hansen S, et al. The combined effect of Parathyroid hormone (1-34) and whole-body Vibration exercise in the treatment of postmenopausal OSteoporosis (PaVOS study): a randomized controlled trial. Osteoporos Int. 2019;30(9):1827-1836.

36. Holmes CA, Ishida W, Elder BD, et al. The effects of highdose parathyroid hormone treatment on fusion outcomes in a rabbit model of posterolateral lumbar spinal fusion alone and in combination with bone morphogenetic protein 2 treatment. World Neurosurg. 2018;115:e366-e374.

37. Wagner D, Kamer L, Sawaguchi T, et al. Sacral bone mass distribution assessed by averaged three-dimensional CT models: implications for pathogenesis and treatment of fragility fractures of the sacrum. J Bone Joint Surg Am. 2016; 98(7):584-590.

38. Elder BD, Ishida W, Lo SL, et al. Use of S2-alar-iliac screws associated with less complications than iliac screws in adult lumbosacropelvic fixation. Spine (Phila Pa 1976). 2017;42(3): E142-E149.

39. O'Brien JR, Yu WD, Bhatnagar R, et al. An anatomic study of the S2 iliac technique for lumbopelvic screw placement. Spine (Phila Pa 1976). 2009;34(12):E439-E442.
40. Lehman RA Jr, Kuklo TR, Belmont PJ Jr, et al. Advantage of pedicle screw fixation directed into the apex of the sacral promontory over bicortical fixation: a biomechanical analysis. Spine (Phila Pa 1976). 2002;27(8):806-811.

41. Keaveny TM, Hoffmann PF, Singh M, et al. Femoral bone strength and its relation to cortical and trabecular changes after treatment with PTH, alendronate, and their combination as assessed by finite element analysis of quantitative CT scans. J Bone Miner Res. 2008;23(12):1974-1982.

42. Langdahl BL, Libanati C, Crittenden DB, et al. Romosozumab (sclerostin monoclonal antibody) versus teriparatide in postmenopausal women with osteoporosis transitioning from oral bisphosphonate therapy: a randomised, open-label, phase 3 trial. Lancet. 2017;390(10102):1585-1594.

43. Graeff C, Marin F, Petto H, et al. High resolution quantitative computed tomography-based assessment of trabecular microstructure and strength estimates by finite-element analysis of the spine, but not DXA, reflects vertebral fracture status in men with glucocorticoid-induced osteoporosis. Bone. 2013; 52(2):568-577.

\section{Disclosures}

Dr. Fogelson has been a consultant for Medtronic. Dr. Currier receives royalties from DePuy Synthes, Zimmer Biomet, and Wolters Kluwer and has direct stock ownership in Spinology. Dr. Nassr has received clinical or research support from AO Spine NA and Premia Spine for the study described. Dr. Anderson has been a consultant for Amgen, Radius Medical, and Medtronic; has direct stock ownership in Titan Spine; and receives royalties from Regeneration Technologies Inc. Dr. Polly has been a consultant for and holds a patent with SI Bone and has received non-studyrelated clinical or research support from Medtronic and Mizuho OSI.

\section{Author Contributions}

Conception and design: Elder, Flanigan, Mikula, Peters, Oushy. Acquisition of data: Elder, Flanigan, Mikula, Peters, Oushy. Analysis and interpretation of data: Elder, Flanigan, Mikula, Peters, Oushy, Anderson. Drafting the article: Elder, Flanigan, Mikula, Fogelson, Bydon, Freedman, Sebastian, Currier, Nassr, Kennel, Anderson, Polly. Critically revising the article: Elder, Flanigan, Mikula, Oushy, Fogelson, Bydon, Freedman, Sebastian, Currier, Nassr, Kennel, Anderson, Polly. Reviewed submitted version of manuscript: Elder, Flanigan, Mikula, Oushy, Fogelson, Bydon, Freedman, Sebastian, Currier, Nassr, Kennel, Polly. Approved the final version of the manuscript on behalf of all authors: Elder. Statistical analysis: Flanigan, Mikula, Oushy. Administrative/technical/material support: Elder. Study supervision: Elder, Flanigan, Mikula, Fogelson, Bydon, Freedman, Sebastian, Currier, Nassr, Kennel, Anderson, Polly.

\section{Supplemental Information}

\section{Previous Presentations}

This work was presented in part as an oral presentation at the Spine Summit 2020 held in Las Vegas, Nevada, on March 5-8, 2020.

\section{Correspondence}

Benjamin D. Elder: Mayo Clinic, Rochester, MN. elder. benjamin@mayo.edu. 